\title{
Downregulation of RdDM during strawberry fruit ripening
}

Jingfei Cheng ${ }^{1,2+}$, Qingfeng Niu ${ }^{2,3+}$, Bo Zhang ${ }^{4}$, Kunsong Chen ${ }^{4}$, Ruihua Yang ${ }^{5}$, Jian-Kang Zhu ${ }^{2,3,6}$,

Yijing Zhang ${ }^{1,2^{*}}$ and Zhaobo Lang ${ }^{2,3^{*}}$ (D)

\begin{abstract}
Background: Recently, DNA methylation was proposed to regulate fleshy fruit ripening. Fleshy fruits can be distinguished by their ripening process as climacteric fruits, such as tomatoes, or non-climacteric fruits, such as strawberries. Tomatoes undergo a global decrease in DNA methylation during ripening, due to increased expression of a DNA demethylase gene. The dynamics and biological relevance of DNA methylation during the ripening of non-climacteric fruits are unknown.

Results: Here, we generate single-base resolution maps of the DNA methylome in immature and ripe strawberry. We observe an overall loss of DNA methylation during strawberry fruit ripening. Thus, ripening-induced DNA hypomethylation occurs not only in climacteric fruit, but also in non-climacteric fruit. Application of a DNA methylation inhibitor causes an early ripening phenotype, suggesting that DNA hypomethylation is important for strawberry fruit ripening. The mechanisms underlying DNA hypomethylation during the ripening of tomato and strawberry are distinct. Unlike in tomatoes, DNA demethylase genes are not upregulated during the ripening of strawberries. Instead, genes involved in RNA-directed DNA methylation are downregulated during strawberry ripening. Further, ripening-induced DNA hypomethylation is associated with decreased siRNA levels, consistent with reduced RdDM activity. Therefore, we propose that a downregulation of RdDM contributes to DNA hypomethylation during strawberry ripening.
\end{abstract}

Conclusions: Our findings provide new insight into the DNA methylation dynamics during the ripening of nonclimacteric fruit and suggest a novel function of RdDM in regulating an important process in plant development.

Keywords: DNA methylation, Fruit ripening, Strawberry, RdDM, siRNA

\section{Background}

Methyl-cytosine $(\mathrm{mC})$ is a conserved epigenetic mark in many eukaryotic organisms and is involved in a wide range of biological processes, such as gene regulation, immunity, imprinting, and genome stability $[1,2]$. Methylation of cytosine occurs in three sequence contexts: symmetric CG, $\mathrm{CHG}$, and asymmetric $\mathrm{CHH}(\mathrm{H}=\mathrm{A}, \mathrm{C}$, or T). In Arabidopsis, DNA methylation in CG and CHG can be maintained by METHYLTRANSFERASE 1 (MET1) and CHROMOMETHYLASE 3 (CMT3) respectively, while $\mathrm{CHH}$ methylation is mainly maintained by CMT2

\footnotetext{
* Correspondence: zhangyijing@sibs.ac.cn; zblang@sibs.ac.cn

${ }^{\dagger}$ Jingfei Cheng and Qingfeng Niu contributed equally to this work.

${ }^{1}$ National Key Laboratory of Plant Molecular Genetics, CAS Center of

Excellence in Molecular Plant Sciences, Institute of Plant Physiology and

Ecology, Shanghai Institutes for Biological Sciences, Chinese Academy of Sciences, Shanghai 200032, China

${ }^{2}$ University of the Chinese Academy of Sciences, Beijing 100049, China

Full list of author information is available at the end of the article
}

$[2,3]$. De novo DNA methylation in all three contexts can be established by DOMAINS REARRANGED METHYLASEs (DRMs) through the RNA-directed DNA methylation (RdDM) pathway. In $\mathrm{RdDM}$, there are two main steps: siRNA biogenesis and siRNA-guided DNA methylation. Pol IV (RNA polymerase IV) and DCLs (DICER-LIKES) are involved in the first step, while Pol V, AGO4/6 (ARGONAUTE 4/6), and DRMs are involved in the second step $[4,5]$. DNA methylation status can be dynamically regulated by both DNA methyltransferases and demethylases. The DNA demethylases, REPRESSOR OF SILENCING 1 (ROS1) family proteins, have DNA glycosylase/lyase activities and thus can actively initiate DNA demethylation process by removal of the $\mathrm{mC}$ base and cleavage of the DNA backbone at the abasic site, leaving a single-nucleotide gap that is later filled with a non-methylated cytosine $[1,6]$.

(c) The Author(s). 2018 Open Access This article is distributed under the terms of the Creative Commons Attribution 4.0 International License (http://creativecommons.org/licenses/by/4.0/), which permits unrestricted use, distribution, and 
Fruit, the angiosperm-specific developmental structure that facilitates seed dispersal, constitutes an important source of human daily diet. The major fruit types include dry fruits, such as nuts, and fleshy fruits, such as peaches. The development of fleshy fruit has three main stages: cell division, cell expansion, and ripening. Fleshy fruits can be further classified as climacteric and non-climacteric, based on their ripening process. The ripening of climacteric fruits, such as tomatoes, is accompanied with increasing ethylene production and respiration bursts, while the ripening of non-climacteric fruits is not [7]. Most studies about ripening mechanisms have been carried out in tomato, a classical climacteric fruit. For the study of non-climacteric fruits, strawberry has been used as a model plant. The ripening of climacteric fruits mainly depends on the phytohormone ethylene. In contrast, the phytohormone abscisic acid (ABA) plays a more prominent role in the ripening of non-climacteric fruits, such as strawberries. Indeed, suppression of 9-cis-epoxycarotenoid dioxygenase (NCED), a vital enzyme in ABA biosynthesis, can cause a severe delay in strawberry ripening [8].

Emerging evidence suggests that DNA methylation also plays an important role in fleshy fruit ripening. In tomato, a naturally occurring epimutation with hypermethylation in the COLORLESS NON-RIPENING (CNR) promoter results in abnormal fruit ripening [9]. In apple, DNA methylation of the $M d M Y B 10$ promoter regulates gene expression and fruit pigmentation during ripening $[10,11]$. In addition, tomatoes undergo global DNA hypomethylation during ripening due to the increased expression of a DNA demethylase gene, and mutations in the DNA demethylase can inhibit ripening [12-14]. Genome-wide DNA methylation dynamics have not been investigated in non-climacteric fruits.

Technological advances have enabled the sequencing and assembly of the genomes for many plant species, including that of the diploid woodland strawberry (Fragaria vesca) [15]. In addition, whole-genome bisulfite sequencing has facilitated the analysis of genome-wide DNA methylation profiles [16], and the DNA methylomes of 34 flowering plants, including leaf tissue from the diploid woodland strawberry, were recently reported [17]. Given that strawberry is an economically important crop, the DNA methylome of strawberry fruit, especially the DNA methylation dynamics during ripening, is of interest. To investigate the epigenetic regulation of non-climacteric fruit ripening, we characterized the DNA methylomes, genome-wide siRNA profiles, and transcriptomes of octoploid cultivated strawberry fruit (Fragaria $x$ ananassa) at different stages of ripening. We observed global DNA demethylation in ripe fruit compared to immature fruit, similar to that observed during tomato ripening. The application of a DNA methylation inhibitor caused hypomethylation and early ripening, suggesting that the ripening-induced decrease in DNA methylation is important for the normal fruit ripening process. We discovered that DNA hypomethylation during strawberry ripening is associated with decreased expression of genes encoding components in the RNA-directed DNA methylation (RdDM) pathway and with decreased siRNA levels. The Tobacco rattle virus (TRV)-induced gene silencing of an important RdDM component, FvAGO4, leads to an early ripening phenotype in strawberry fruit. It is noteworthy that hundreds of ripening-regulated genes display DNA hypomethylation in their promoters, which helps to explain the epigenetic regulation of strawberry fruit ripening. In summary, our study for the first time reveals the dynamics of DNA methylation in non-climacteric fruit ripening and suggests a distinct mechanism for the loss of DNA methylation during fruit ripening.

\section{Results}

\section{The DNA methylome of strawberry fruit}

To characterize strawberry methylomes, we performed whole-genome bisulfite sequencing and generated single-base resolution maps of DNA methylation for both leaf and fruit tissues of $F$. ananassa. Fruits from immature to ripe stages (Fa1-Fa3) were sequenced with two biological replicates (Fig. 1a). Due to the high level of collinearity between cultivated octoploid strawberry (F. ananassa) and wild diploid strawberry (F. vesca), and the lack of a well-assembled genome for $F$. ananassa, we used the genome of $F$. vesca as reference $[15,18]$ in our analyses. The genome of $F$. vesca is about $240 \mathrm{MB}(2 n=$ 14). For each sequencing library, at least $100 \mathrm{M}$ paired-end reads (read length $=150 \mathrm{bp}$ ) were produced, covering $>80 \%$ of the genome. For each bisulfite-treated library, $\sim 10 \%$ of the total reads were mapped to the unmethylated chloroplast genome, and the conversion rates were $>99.6 \%$ for all libraries (Additional file 1: Table S1). Each methylome was sequenced with an average $>10$-fold coverage per DNA strand. The sequencing coverage and depth are comparable to those of published methylomes of Arabidopsis and tomato [14, 19].

Immature strawberry fruit displayed an average DNA methylation of about 7.5\%, which is lower than that of tomato fruit (22\%) [14], and the average methylation levels of $\mathrm{mCG}, \mathrm{mCHG}$, and $\mathrm{mCHH}$ were 40,11 , and $2 \%$, respectively. In plants, transposable elements (TEs) and repeats are usually highly methylated. To investigate the genomic distribution of DNA methylation in strawberry, we annotated TEs and repeats in the $F$. vesca genome de novo using RepeatScout [20]. TE and repeat contents in the strawberry genome were higher than those of Arabidopsis, but lower than those of rice and tomato (Additional file 2: Figure S1a), consistent with proposals that 

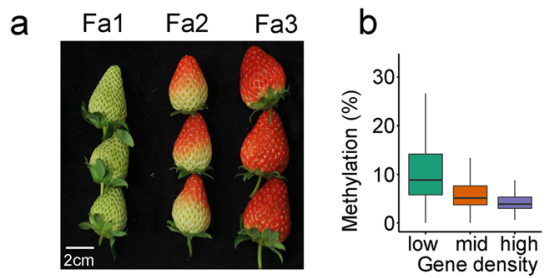

d
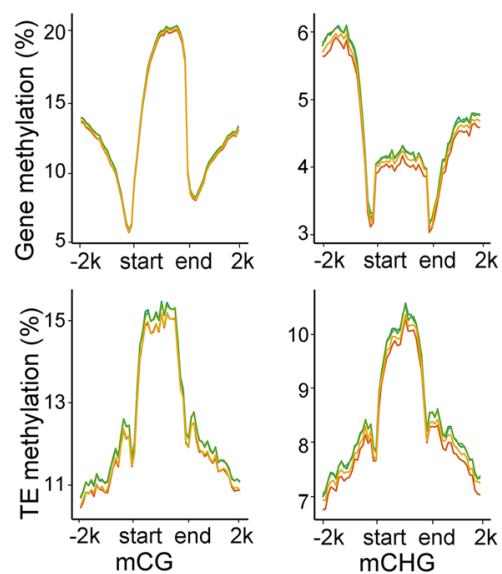

C
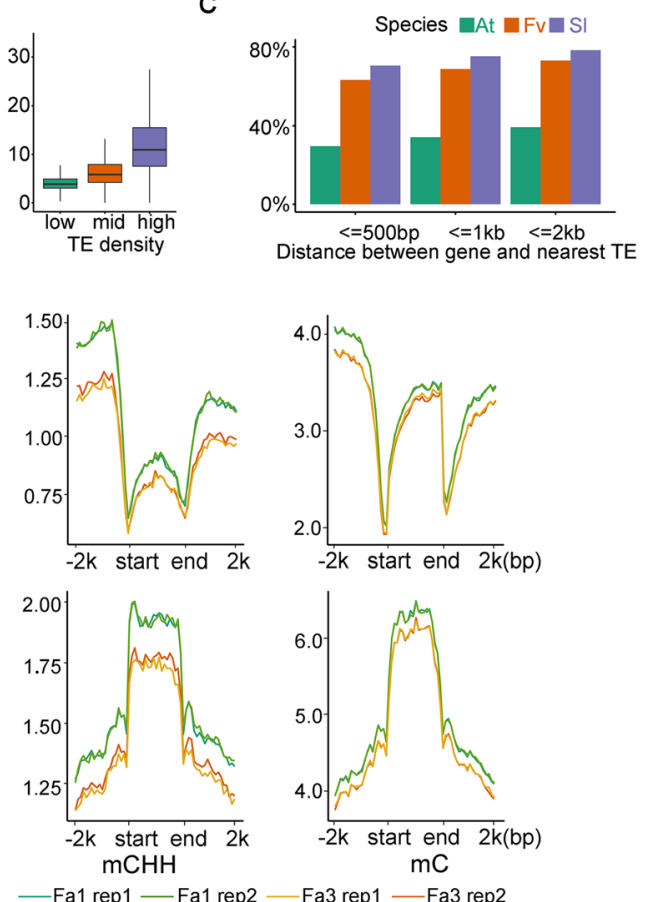

Fig. 1 Characterization of strawberry methylomes. a Picture of strawberry fruits at different stages. Immature (Fa1), half-red (Fa2), and fully red (Fa3) fruits were used. $\mathbf{b}$ Correlation between DNA methylation level and gene (left panel) or TE densities (right panel). c Percentages of genes that have TEs within $500 \mathrm{bp}, 1 \mathrm{~kb}$ and $2 \mathrm{~kb}$ in Arabidopsis (At), strawbern (Fv), and tomato (Sl) genomes, respectively. d DNA methylation profiles of $\mathrm{mCG}, \mathrm{mCHG}, \mathrm{mCHH}$ and mC surrounding genes (upper panel) and TEs (lower panel) in immature (Fa1) and fully red (Fa3) fruits. Two biological replicates were shown. Transcription start site (TSS) and transcription end site (TES) are indicated

larger genomes tend to have higher repeat contents [21]. Further, TEs and repeats, as well as DNA methylation, in the strawberry genome were not obviously concentrated in pericentromeric regions (Additional file 2: Figure S1b), unlike in Arabidopsis and tomato genomes, possibly because the strawberry genome is not as well-assembled as the Arabidopsis and tomato genomes, particularly in the pericentromeric regions. However, similar to Arabidopsis and tomato, DNA methylation was high in TE- and repeat-rich genomic regions, and low in gene-rich regions in strawberry fruit (Fig. 1b). TEs and repeats were methylated in all three contexts, whereas gene bodies were enriched with mCG but depleted of non-CG methylation (Additional file 2: Figure S1c).

Leaves displayed an average $\mathrm{mC}$ level of about $8 \%$, slightly higher than that of immature fruits (7.5\%). Leaves and fruit exhibited similar DNA methylation patterns around genes and TEs, but in general, leaves have a higher DNA methylation level relative to fruits (Additional file 2: Figure S1c). According to a previous study, genes in medium-sized genomes, such as tomato, have shorter distances to the nearest TEs compared to those in small genomes such as Arabidopsis and thus are more likely regulated by methylation changes of nearby TEs [14]. The distances between genes and the closest TEs in the strawberry genome are shorter than that in Arabidopsis, but longer than that in tomato (Fig. 1c), suggesting that strawberry genes might be more vulnerable to nearby methylation changes than Arabidopsis genes.

\section{Loss of DNA methylation during strawberry ripening}

DNA methylation decreases dramatically during the ripening of tomato, a typical climacteric fruit. To investigate DNA methylation dynamics during ripening of a non-climacteric fruit, we compared the DNA methylomes of strawberry fruit at three different stages (Fa1-Fa3), with two biological replicates for each stage (Fig. 1a). Principal component analysis (PCA) showed consistency between two biological replicates at each stage (Additional file 2: Figure S2a). We found that, in general, ripe fruits have a lower DNA methylation level than immature fruits around genes and TEs (Fig. 1d), suggesting a decrease in DNA methylation during ripening. This decrease occurred in both biological replicates (Fig. 1d). To identify ripeninginduced differentially methylated regions (DMRs), we compared the methylomes of $\mathrm{Fa} 1$ and $\mathrm{Fa} 3$ fruits. Because the two biological replicates were highly consistent, we combined the data from the two biological replicates to increase the statistical power of DMR calling. We found a total of 2766 DMRs in Fa3 compared to Fa1, among which 
466 were hypermethylated (hyper-DMRs) and 2300 were hypomethylated (hypo-DMRs) (Additional file 3: Table S2). The DNA methylation changes showed a high correlation between biological replicates (cor $=0.58$, Additional file 2: Figure S2b). The larger number of hypo-DMRs vs. hyper-DMRs also suggests a decrease in DNA methylation during strawberry ripening. To investigate whether the change of methylation preferentially occurred in any sequence context, we examined DNA methylation levels of hypo-DMRs and hyper-DMRs in the two biological replicates in CG, CHG, and $\mathrm{CHH}$, respectively. As shown in Fig. 2a and Additional file 2: Figure S2c, the two biological replicates showed the same trend of change that DNA methylation was gradually changed in all three contexts from Fa1 to Fa3 (Fig. 2a, Additional file 2: Figure S2c). The methylation levels of several representative hypo- and hyper-DMRs are shown in Fig. 2b, Additional file 2: Figure S2d-e. We calculated the DNA methylation levels for each cytosine in all three contexts within all DMRs, which showed a left-skewed pattern, suggesting that most cytosines were hypomethylated in Fa3 compared to $\mathrm{Fa} 1$ (Fig. 2c). Together, these analyses suggest that strawberries undergo an overall loss of DNA methylation during ripening, similar to tomatoes [12].

To investigate the importance of the decreased DNA methylation during ripening, we treated young fruits with a DNA methylation inhibitor, 5-azacytidine. As shown in Fig. 3a, compared with mock treatment, 5-azacytidinetreated fruits exhibited an early ripening phenotype. To examine whether the 5-azacytidine treatment influenced fruit ripening through altering DNA methylation level in the fruits, we performed methylation-sensitive qPCR for both mock- and 5-azacytidine-treated fruits. At the two examined genomic regions, 5-azacytidine-treated fruits showed decreased DNA methylation levels compared to mock-treated fruits (Fig. 3b). These results suggest that the DNA hypomethylation is important for strawberry fruit ripening.

Next, we evaluated the genomic distribution of DMRs. We found that hypo-DMRs were enriched at genomic regions encompassing transcriptional start sites (TSS) (Fig. 3c, d). In particular, the distribution of DMRs around genes revealed that hypo-DMRs, especially at $\mathrm{mCHH}$, were enriched at the $5^{\prime}$ - and 3 '-flanking regions of genes (Fig. 3e, f). In contrast, the distribution of hyper-DMRs did not show such a pattern (Fig. 3f, Additional file 2: Figure S3). These results suggest that ripening-induced DNA hypomethylation potentially regulates gene expression.

\section{Reduced expression of RdDM pathway genes during ripening}

The DNA hypomethylation during tomato fruit ripening is due to increased expression of the DNA demethylase gene, SIDML2 [14]. To investigate the mechanism of DNA hypomethylation during strawberry ripening, we first examined the expression of DNA demethylase genes. We generated genome-wide transcript profiles for strawberry fruit at Fa1-Fa3 stages shown in Fig. 1a, with two biological replicates at each stage that showed good consistency via PCA (Additional file 2: Figure S4a). In Arabidopsis, DNA demethylation is initiated by the REPRESSOR OF SILENCING (ROS1) family of glycosylase/lyase proteins, which can remove the $\mathrm{mC}$ base [1]. The strawberry genome harbors 4 ROS1 homologs, including gene01635 (FvDME1), gene30143 (FvROS1.1), gene30462 (FvROS1.2), and gene11785 (FvROS1.3) (Additional file 2: Figure S4b). None of these ROS1 homologs showed significantly increased expression during ripening (Additional file 2: Figure S4c). The IDM (Increase of DNA methylation) protein complex can regulate ROS1 targeting and, in turn, DNA demethylation in Arabidopsis [22, 23]. We examined the expression of strawberry genes that are similar to the AtIDMs, including gene27227 (FvIDM1), gene21977 (FvIDM2), and gene31466 (FvIDM3) (Additional file 2: Figure S4b). However, none of these was upregulated during ripening (Additional file 2: Figure S4c). These results suggest that DNA hypomethylation during strawberry ripening is not associated with increased expression of DNA demethylation pathway genes.

DNA demethylation and DNA methylation can antagonize each other to dynamically regulate the plant methylome. We hypothesized that a reduced DNA methylation activity could contribute to the DNA hypomethylation during strawberry fruit ripening. To test this hypothesis, we examined the expression of genes involved in DNA methylation pathways. In Arabidopsis, mCG, $\mathrm{mCHG}$, and $\mathrm{mCHH}$ are maintained by METHYLTRANSFERASE 1 (MET1), CHROMOMETHYLASE 3 (CMT3), and CMT2 and DOMAINS REARRANGED METHYLASEs (DRMs), respectively, whereas all three contexts can be de novo methylated by DRMs via the RNA-directed DNA methylation (RdDM) pathway $[1,2]$. We identified 8 DNA methyltransferase genes in the strawberry genome (annotated in Phytozome), including orthologs to AtMET1 (gene13037 (FvMET1)), AtCMTs (gene13664 (FvCMT2), gene10077 (FvCMT3.1), and gene15171 (FvCMT3.2)), and AtDRMs (gene05866 (FvDRM1.1), gene06047 (FvDRM1.2), gene28439 (FvDRM1.3) and gene17910 (FvDRM3.1)) (Fig. 4a). We found that FvDRM1.1 was barely expressed in fruits $(\mathrm{FPKM}<2)($ data not shown) and FvMET1, FvCMT2, and $F v D R M 1.2$ were not differentially expressed during ripening (adjusted $P$ value $<0.05$ ) (Fig. 4b, Additional file 4: Table S3). However, FvCMT3.1, FvCMT3.2, FvDRM1.3, and $F v D R M 3.1$ were all downregulated during ripening (Fig. 4b).

The downregulation of FvDRM1.3 and FvDRM3.1 suggested that RdDM activity may be reduced during strawberry ripening. To further investigate whether $\operatorname{RdDM}$ 
a
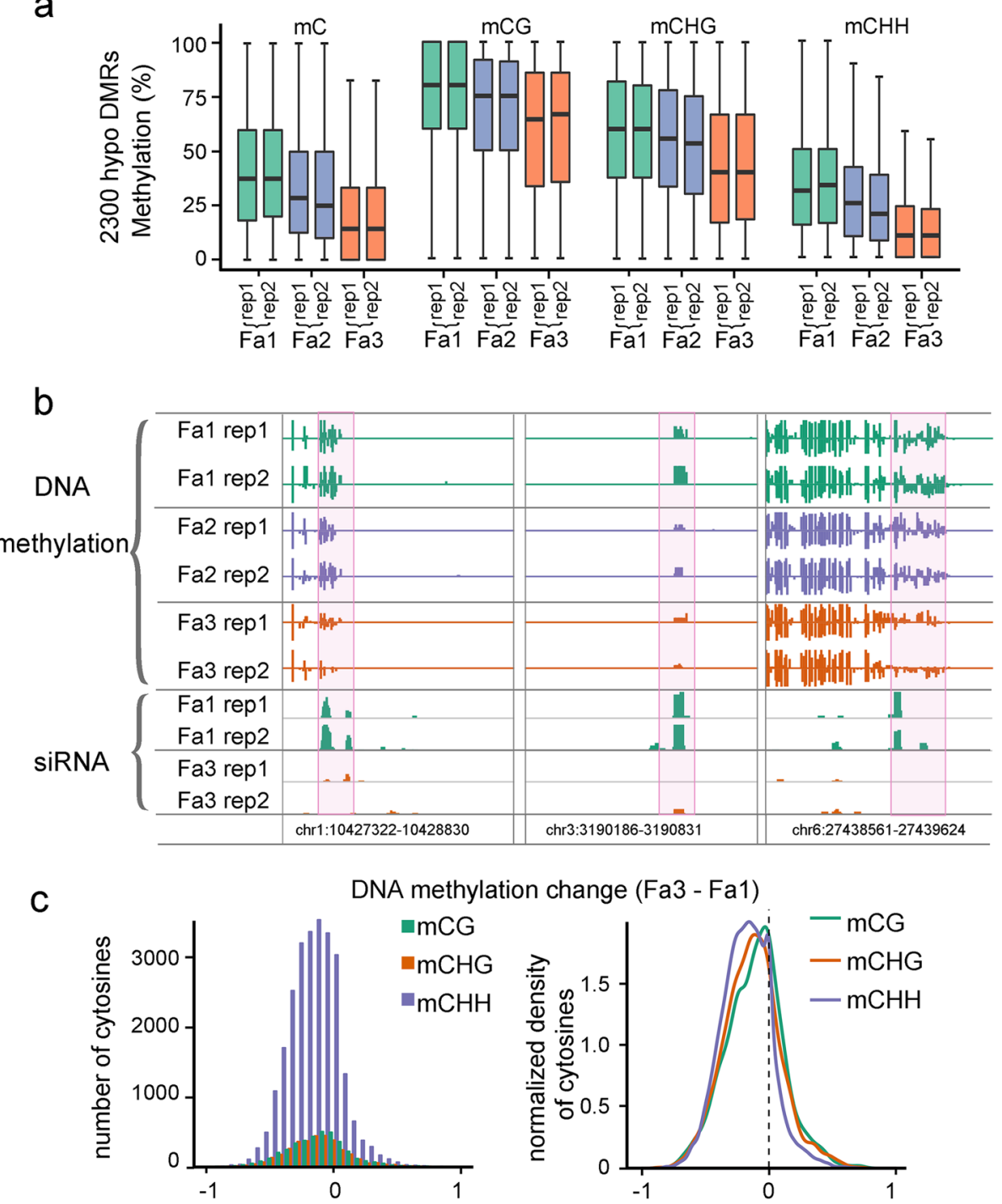

Fig. 2 DNA methylation dynamics during strawberry ripening. a Boxplots showing DNA methylation levels of 2300 hypo-differentially methylated regions (DMRs) in Fa3 relative to Fa1 in all stages. Methylation levels in $\mathrm{mC}, \mathrm{mCG}, \mathrm{mCHG}$, and $\mathrm{mCHH}$ contexts of two biological replicates are shown. b Integrated Genome Browser (IGB) display of DNA methylation levels and 24-nt siRNA levels of representative hypo-DMRs. DNA methylation levels of cytosines and siRNA levels are indicated by the heights of the vertical bars on each track. Genome coordinates are indicated at the bottom. Two biological replicates are shown. c Distribution of ripening-induced methylation change of cytosines in different contexts. Cytosines within 2766 DMRs in Fa3 relative to Fa1 were used. DNA methylation change (Fa3-Fa1) for each cytosine was plotted according to the number of cytosines

activity is reduced during strawberry ripening, we examined the expression of other RdDM components. RdDM involves two steps, siRNA biogenesis, which requires Pol IV, RDR2, and DCL3, and siRNA-guided DNA methylation, which requires Pol V, AGO4/6, and DRMs [5] (Fig. 4c). We identified strawberry orthologs of Pol IV and Pol V largest subunits, RDR2, DCL3, AGO4, and $A G O 6$, and found that all of these genes displayed reduced transcript levels from Fa1 to Fa3 (adjusted $P$ value $<0.05)$. The expression patterns of these genes were confirmed by analysis of published transcriptome data (see methods for detailed information) (Additional file 2:
Figure S4d). The decreased expression of FvCMTs as well as FvDRMs and other RdDM pathway genes is consistent with the observed loss of DNA methylation during ripening, especially the loss of non-CG methylation. Together, these data suggest that reduced DNA methylation activities contribute to ripening-induced DNA hypomethylation.

siRNAs are diminished at ripening-induced hypo-DMRs The decreased expression of genes involved in RdDM during ripening (Fig. 4) suggests that RdDM activity is reduced. To 

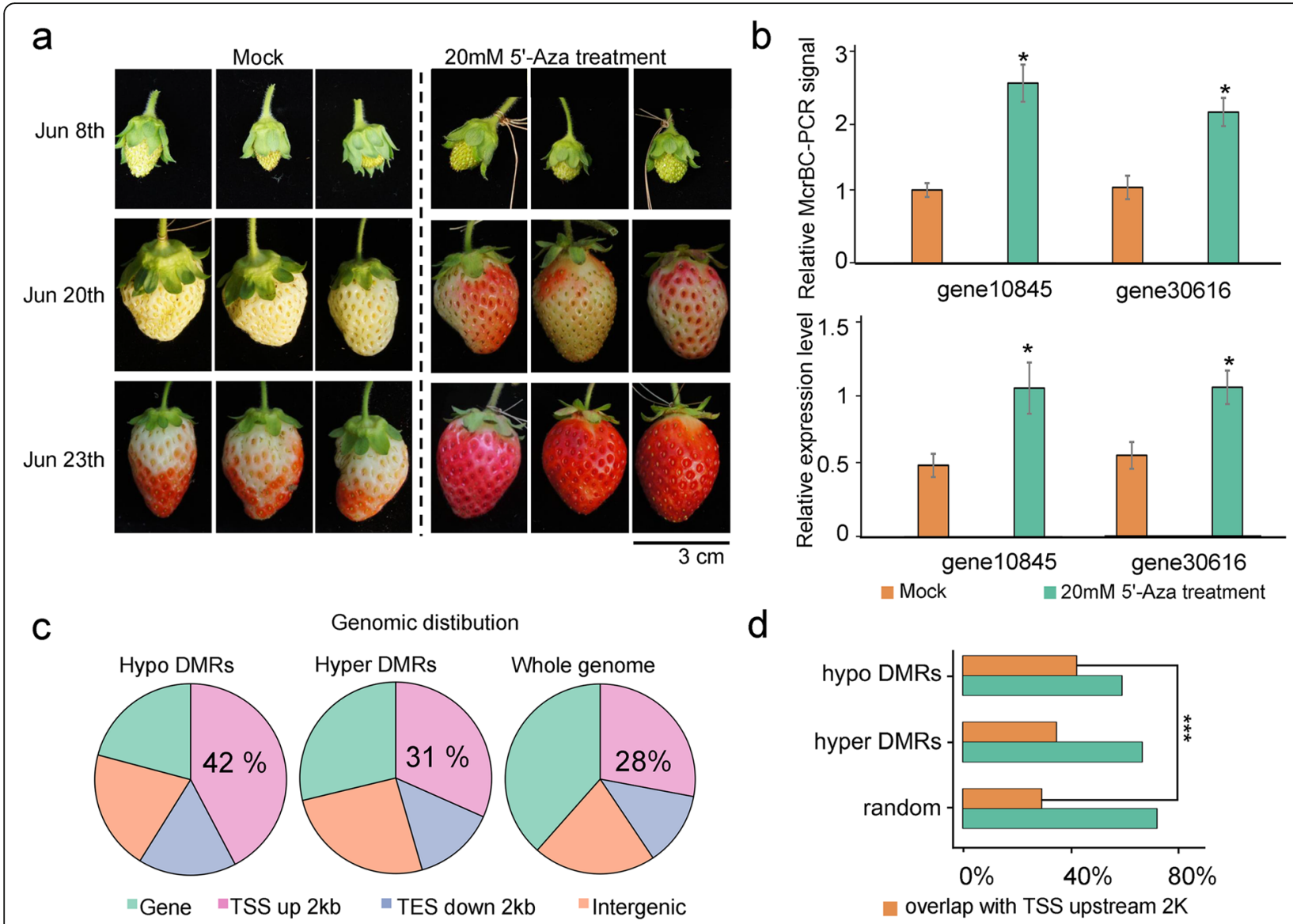

d

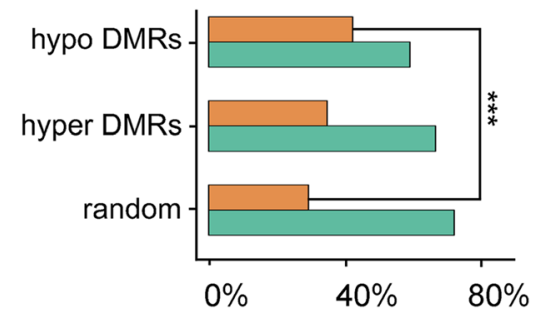

overlap with TSS upstream $2 \mathrm{~K}$

no overlap with TSS upstream $2 \mathrm{~K}$

e
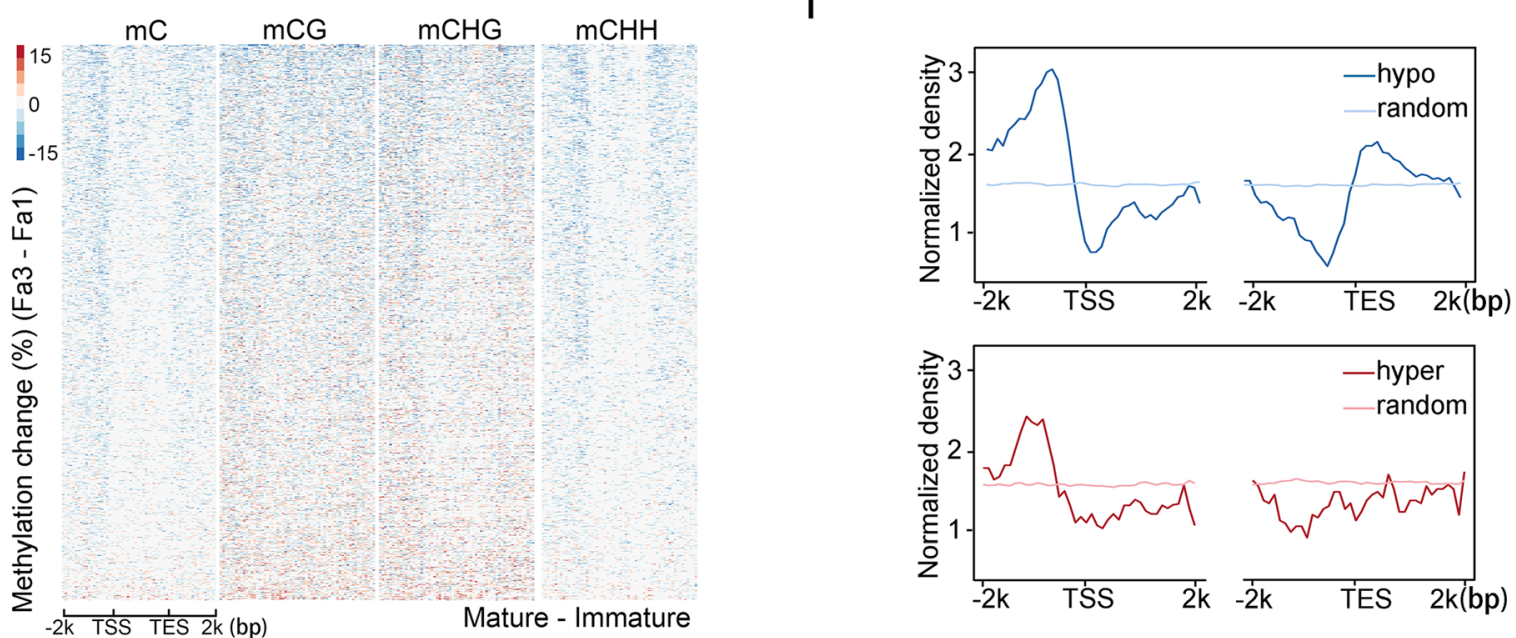

Fig. 3 Characterization of ripening-induced DNA hypomethylation. a Pictures of DNA methylation inhibitor 5-azacytidine-treated fruits. Fruits treated with water served as control (mock). b McrBC-qPCR analysis of DNA methylation levels (upper panel) and qPCR analysis of gene expression levels (lower panel) of two genes in mock and 5-azacytidine-treated fruits. In McrBC-qPCR analysis, a higher qPCR signal indicates lower $m C$ level. Error bars indicate SD, $n=3$ ( ${ }^{*}$ v value $<0.05$, two-tailed $t$ test). c Distribution of genomic elements within ripening-induced hypo-DMRs, hyper-DMRs, and the whole genome. Gene, gene body; TSS up 2 kb, 2 kb upstream of TSS; TES down 2 kb, 2 kb downstream of TES; Intergenic, intergenic regions. d Hypo-DMRs are significantly associated with regions $2 \mathrm{~kb}$ upstream of TSS as compared to random genomic regions ( ${ }^{* * *} P$ value $<0.01$, as determined using Fisher's exact test). e Heatmaps showing DNA methylation changes (Fa3-Fa1) across hypo-DMR-associated genes. Methylation changes in mC, mCG, mCHG, and mCHH contexts are shown. $\mathbf{f}$ Distributions of hypo-DMRs (blue line in upper panel) and hyper-DMRs (red line in lower panel) around genes as compared to that of randomly selected genomic regions (light-blue line and light-red line) 


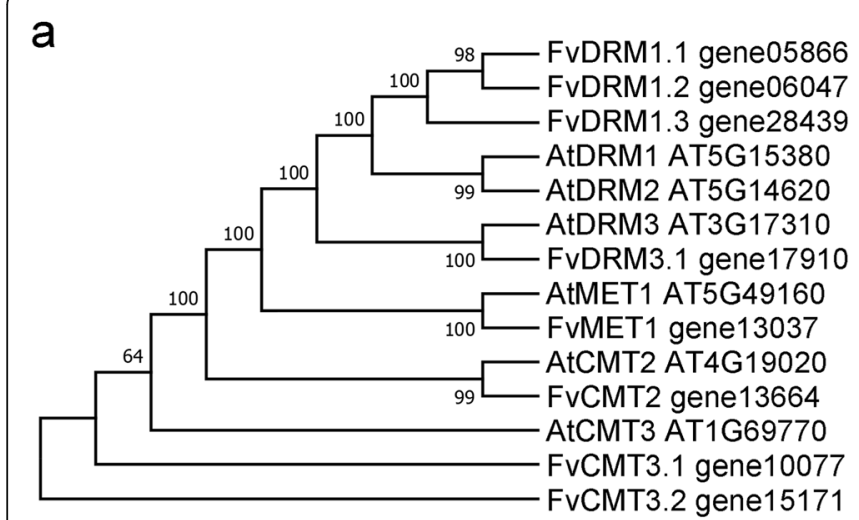

C
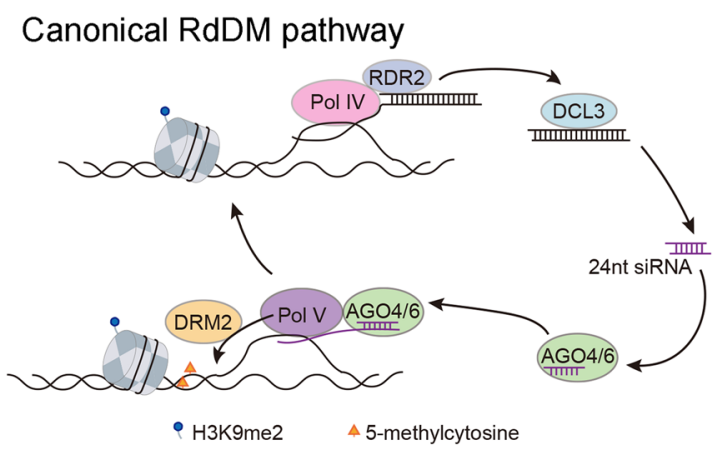

b

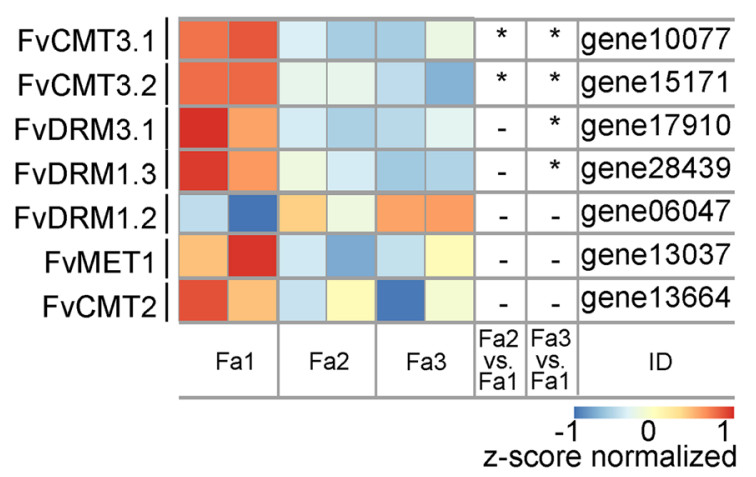

Expression of genes involved in RdDM

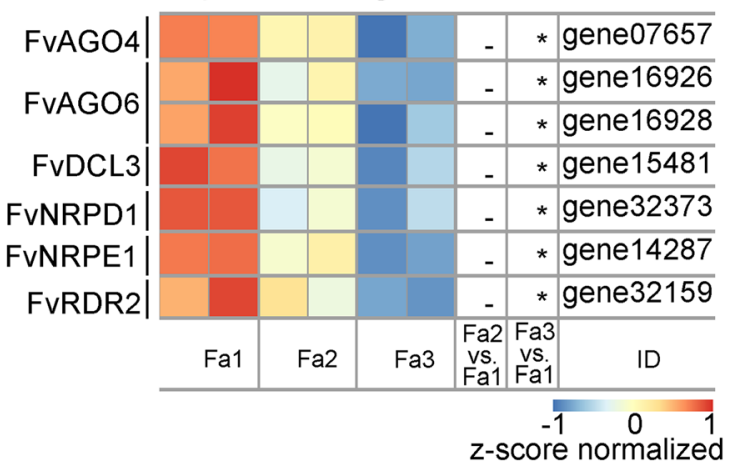

Fig. 4 Expression of genes involved in DNA methylation. a Phylogenetic analysis of DNA methyltransferase genes in strawberry and Arabidopsis. $\mathbf{b}$ Heatmap showing transcript levels of DNA methyltransferase genes in Fa1 Fa3 (*adjusted $P$ value $<0.05$, as determined using the DESeq). c Transcript levels of genes involved in the RdDM pathway in Fa1 Fa3. A brief working model of RdDM pathway is shown on the left. Pol IV (RNA polymerase IV), RDR2 (RNA-Dependent RNA polymerase 2), and DCLs (DICER-LIKES) are required for siRNA biogenesis; Pol V, AGO4/6 (ARGONAUTE 4/6), and DRM2 are involved in siRNA-guided DNA methylation. NRPD1 and NRPE1 are the largest subunits of Pol IV and Pol V respectively. Heatmap (right panel) shows transcript levels of genes involved in RdDM pathways (* adjusted $P$ value $<0.05$, as determined using the DESeq)

assess RdDM activity more directly, we examined the levels of RdDM-dependent 24-nt siRNAs [2].

First, we used deep sequencing to evaluate the genome-wide siRNA profiles in Fa1 and Fa3. Consistent with the small RNA composition in Arabidopsis, 21-nt and 24-nt small RNAs represented the most abundant small RNAs in all sequenced samples of strawberry fruits (Fig. 5a). RdDM-dependent 24-nt siRNAs in Arabidopsis are characterized by an adenosine at the $5^{\prime}$-end [24]. We found that 24-nt siRNAs in strawberry also have a preference for $5^{\prime}$ terminal adenosine (Additional file 2: Figure S5a), suggesting that 24-nt siRNA biogenesis is conserved in strawberry and Arabidopsis. Further analysis revealed that strawberry 24-nt siRNAs are preferentially located in promoter and 3 ' regulatory regions around genes, but are more widely spread along TE bodies and flanking regions (Fig. 5b).

The biogenesis of 24-nt siRNA depends on RdDM. However, in Arabidopsis, it is known that long TEs usually have siRNAs in their body regions, even though their body methylation relies on CMT2 [3]. In strawberry, we identified 370,630 24-nt siRNA clusters (Additional file 5: Table S4); 345,828 of these clusters are not located in long TE bodies and thus can be considered as canonical RdDM target regions. We found that over $67 \%$ of ripeninginduced hypo-DMRs overlapped with the siRNA clusters (Fig. 5c). In contrast, only $17 \%$ of randomly selected genomic regions overlapped with the siRNA clusters, suggesting that ripening-induced hypo-DMRs are significantly associated with siRNAs $(p<0.01)$ (Fig. $5 c)$. Further, ripening led to diminished DNA methylation and decreased numbers of siRNA clusters in the bodies and flanking regions of genes and TEs (Fig. 5d, Additional file 2: Figure S5b). To further study the relationship between siRNA-mediated DNA methylation and ripening-induced DNA hypomethylation, we monitored changes in siRNA enrichment at ripening-induced hypo-DMRs. We found that siRNA levels were reduced at ripening-induced hypo-DMRs from Fa1 to Fa3 (Fig. 5e and Additional file 2: Figure S5c). In addition, we observed a decreased $\mathrm{mCHH}$ 

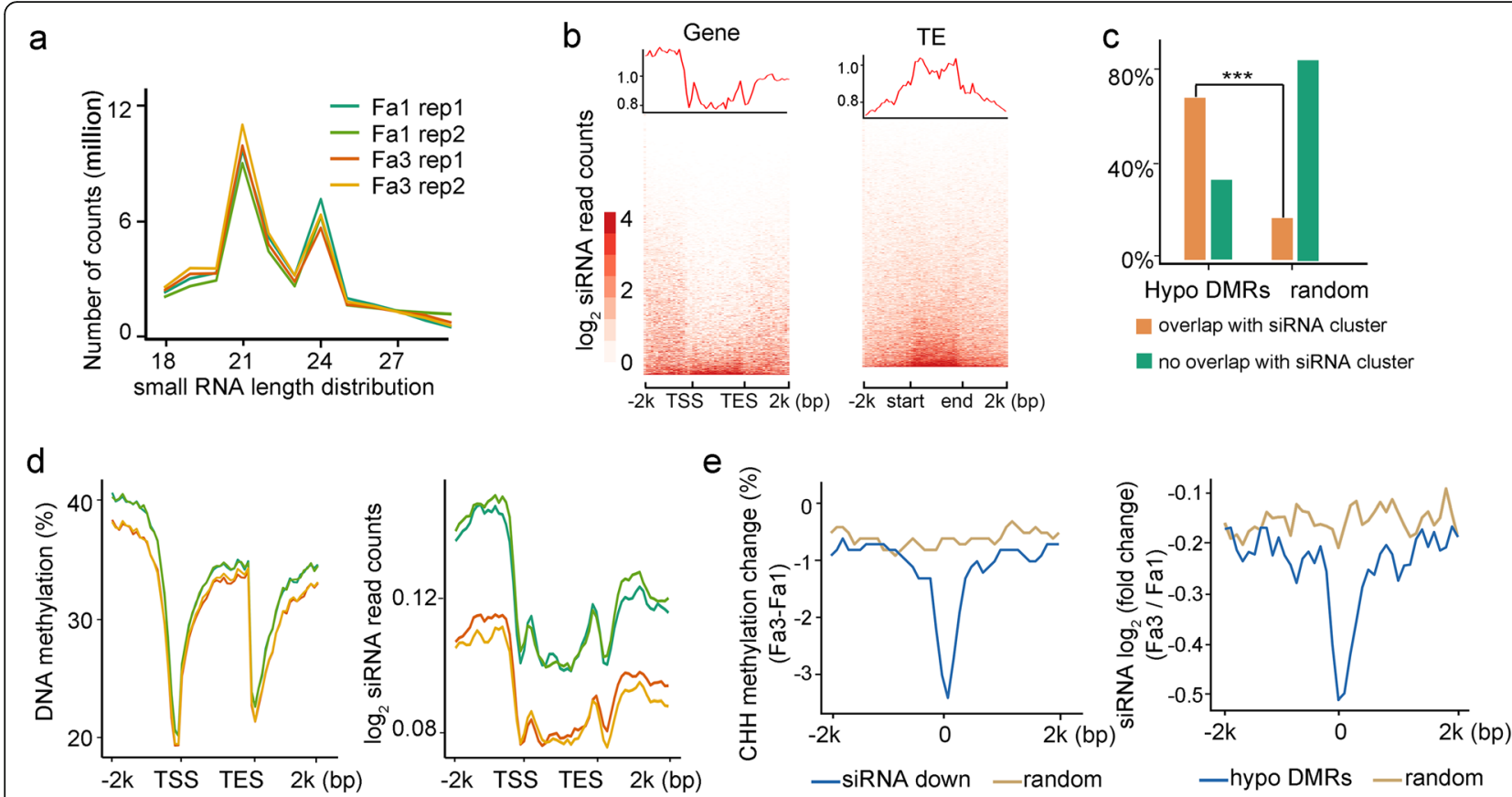

Fig. 5 Association between ripening-induced siRNA decrease and DNA hypomethylation. a Size distribution of sequenced strawberry small RNAs. Two biological replicates of Fa1 and Fa3 are shown. b Average profile and heatmaps showing 24-nt siRNA distribution surrounding genes (left) and TEs (right). c Hypo-DMRs are significantly associated with siRNA clusters as compared to random genomic regions (*** $P$ value $<0.01$, as determined using Fisher's exact test). $\mathbf{d}$ Profiles of DNA methylation (left panel) and siRNA (right panel) surrounding genes in Fa1 and Fa3. Two biological replicates of Fa1 and Fa3 are shown. e Change of CHH methylation surrounding siRNA downregulated regions (left) and change of 24-nt siRNA level surrounding hypo-DMRs (right) on average

level at genomic regions with reduced siRNA levels (Fig. 5e). The siRNA levels at several representative hypo-DMRs are shown in Fig. 2b. To test the significance of RdDM pathway on strawberry ripening, we used TRV-mediated gene silencing to downregulate $F v A G O 4$ in young strawberry fruits. We observed an early ripening phenotype in TRV2:FvAGO4 fruits compared to control TRV2-only fruits (Additional file 2: Figure S5d), consistent with the involvement of $\mathrm{RdDM}$ in strawberry ripening. These analyses suggest that the reduced expression of RdDM pathway genes leads to diminished siRNA accumulation and decreased RdDM activity, thus contributing to DNA hypomethylation and ripening of strawberries.

\section{DNA methylation changes are associated with altered gene expression in fruits}

To investigate the relationship between ripening-induced changes in DNA methylation and gene expression, we compared the transcriptomes for fruits at Fa1 and Fa3. We identified a total of 2316 differentially expressed genes (DEGs, adjusted $P$ value $<0.01$ ), including 899 upregulated DEGs (up-DEGs) and 1417 downregulated DEGs (down-DEGs) in Fa3 relative to Fa1 (Fig. 6a and Additional file 6: Table S5). Next, we examined the DNA methylation levels at the up- and downregulated DEGs. We found that a large subset of the DEGs undergoes a loss of DNA methylation from Fa1 to Fa3, especially in non-CG contexts, at their 5 ' - and $3^{\prime}$-regulatory regions (Fig. 6b-c, Additional file 2: Figure S6a and Additional file 6: Table S5). DNA methylation, especially at promoters, is often associated with transcriptional silencing. For upregulated DEGs, the decrease in DNA methylation corresponded to increased expression during ripening, which is consistent with the role of DNA methylation in gene silencing (Fig. 6c). To investigate the significance of DNA methylation in the regulation of gene expression, we examined the methylation and expression levels of two up-DEGs (gene10875 and gene30616) in mock and 5 -azacytidine-treated samples. Compared with mock treatment, the promoter regions of these two genes were hypomethylated and their expression was upregulated in 5-azacytidine-treated fruits, suggesting a repressive role of DNA methylation for these two genes (Fig. 3b). For downregulated DEGs, the DNA hypomethylation corresponded to decreased expression during ripening (Fig. 6b, c). A recent study using tomato DNA demethylase mutants revealed that DNA demethylation is associated with the repression of several hundred genes during tomato ripening [14]. How DNA demethylation causes gene repression is still unclear in both tomato and strawberry.

Fruit ripening is a complex developmental process that involves numerous physiological, biochemical, and structural 


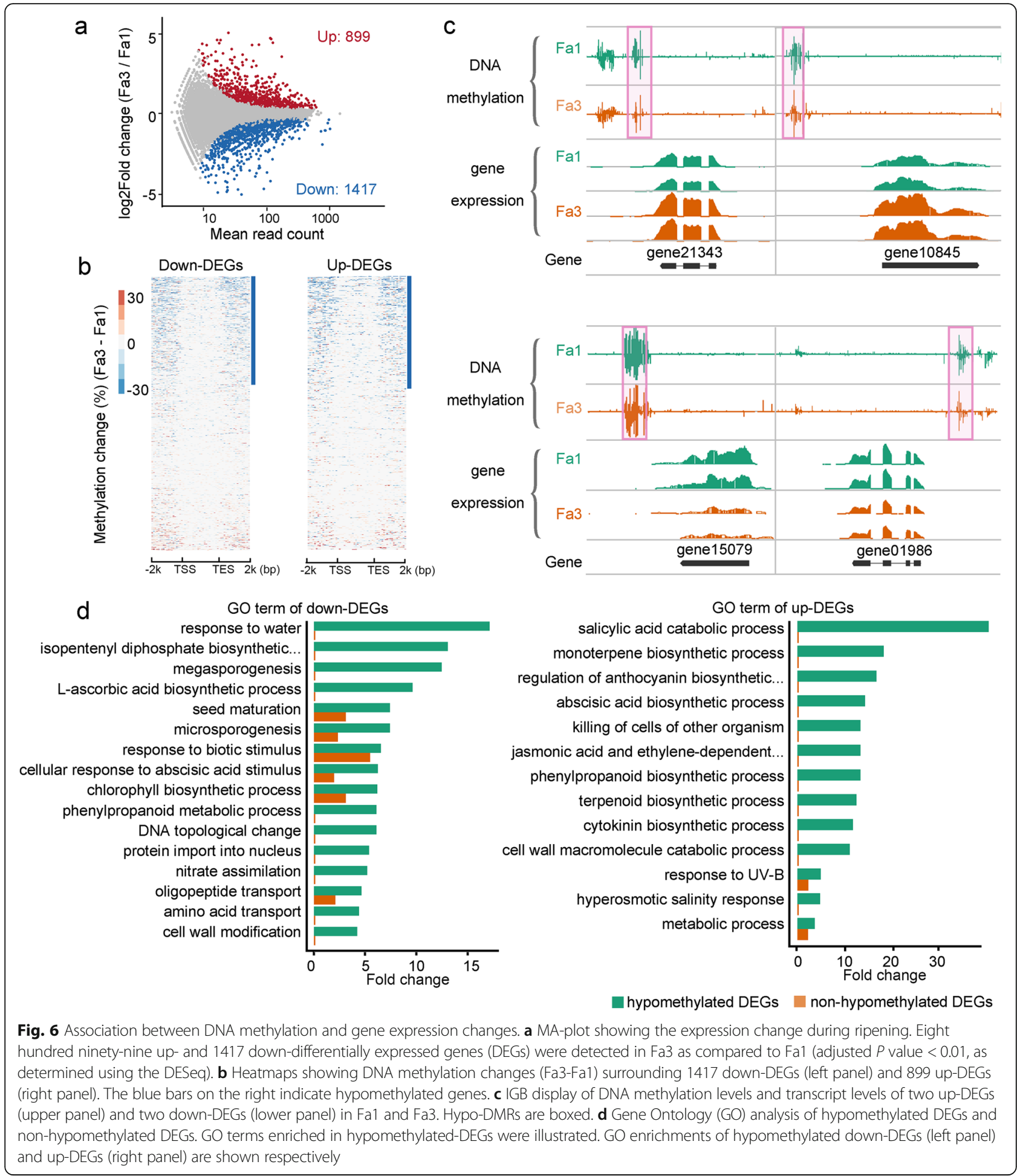

alterations. To understand how DNA methylation-associated gene regulation contributes to the ripening process, we performed Gene Ontology (GO) analysis for hypomethylated up- and downregulated DEGs. Up- and downregulated DEGs that are not hypomethylated served as controls (Fig. 6d). This analysis revealed that genes involved in the "regulation of anthocyanin biosynthetic process" are enriched in upregulated DEGs (Fig. 6d and Additional file 7: Table S6), suggesting that DNA methylation may regulate fruit coloration during ripening. A previous study found that gene21343 (expansin-A8-like) is required for biosynthesis of the pigment anthocyanin during strawberry ripening [25], 
although the underlying molecular mechanism was unclear. Our results showed that gene21343 displayed decreased DNA methylation during ripening, and its expression was upregulated accordingly (Fig. 6b, Additional file 2: Figure S6b). Cytokinin is important for early fruit development, and a recent study suggested that cytokinin accumulates during the ripening of kiwi, grape, and strawberry fruits [26]. Consistent with these findings, our analysis found that genes involved in the "cytokinin biosynthetic process" are enriched in the upregulated DEGs (Fig. 6d and Additional file 7: Table S6). The endogenous content of ABA is known to increase substantially during strawberry ripening, and the application of an ABA synthesis inhibitor interrupts strawberry ripening [8]. Consistent with these observations, we found that genes involved in the "abscisic acid biosynthetic process" are enriched in the upregulated DEGs (Fig. 6d and Additional file 7: Table S6). Several other ripening-related GO terms were also included in the upregulated DEGs, such as genes involved in the biosynthesis of flavor volatiles (Fig. 6d and Additional file 7: Table S6).

On the other hand, many genes need to be downregulated during ripening. For example, genes involved in photosynthesis are active and required in the early stages of fruit development, but are repressed during ripening to allow conversion of chloroplasts into chromoplasts, which are important for fruit coloration. The hypomethylated downregulated DEGs were enriched for "chlorophyll biosynthetic process," suggesting that DNA hypomethylation may contribute to the decline in photosynthesis during ripening. We also found several GO terms related to cell wall biosynthesis and metabolism in hypomethylated downregulated DEGs. During early fruit development, genes involved in cell wall biosynthesis and modification are active to sustain fast cell division and cell enlargement, but these genes are not needed later during the ripening stage. Interestingly, the ripening-related GO terms were only enriched in hypomethylated DEGs, but not enriched in up- or down-DEGs without hypomethylation (Fig. 6d and Additional file 7: Table S6). Together, these analyses suggest that DNA methylation-mediated gene regulation controls many biological processes important for ripening.

\section{Discussion}

DNA methylation was proposed to be an important factor in the regulation of fruit ripening, mainly based on studies in tomatoes [7]. Tomato, a typical climacteric fruit, undergoes genome-wide loss of DNA methylation during ripening [12]. However, the dynamics and relevance of DNA methylation in non-climacteric fruit ripening, or in any other climacteric fruit, were unknown. Here, we generated single-base resolution maps of DNA methylation for strawberry leaves and fruits at different stages (Fig. 1a). We found that, similar to tomatoes, strawberry fruits undergo an overall loss of DNA methylation, especially in non-CG contexts, during ripening (Fig. 2) [14]. Our results suggest that ripening-induced DNA hypomethylation could occur in both climacteric and non-climacteric fruits.

DNA methylation can be dynamically regulated by DNA methylation and DNA demethylation activities. In Arabidopsis, active DNA demethylation is catalyzed by ROS1 family proteins, which can remove the $\mathrm{mC}$ base and cleave the DNA backbone, leaving a single-nucleotide gap that can be filled with a non-methylated cytosine [1]. The loss of DNA methylation during tomato fruit ripening is caused by increased expression of SIDML2, a tomato ortholog of Arabidopsis ROS1 [14]. However, none of the DNA demethylase genes in strawberry was upregulated during ripening (Additional file 2: Figure S4). Instead, we found that several genes encoding DNA methyltransferases and other key components in the $\mathrm{RdDM}$ pathway were significantly downregulated during strawberry fruit ripening (Fig. 4). We inferred that $\mathrm{RdDM}$ activity is reduced during strawberry ripening, such that DNA demethylation becomes relatively dominant over methylation, leading to an overall loss of DNA methylation. In our model, even though DNA methylation activities are decreasing, the active DNA demethylation machinery must be active during ripening, to remove the existing DNA methylation, since there is little cell division in ripening fruits and thus passive demethylation should be insignificant. Indeed, we found that two of the strawberry DNA demethylase genes were expressed during ripening (FPKM > 20).

RdDM is a well-known DNA methylation pathway that consists of two main steps: siRNA biogenesis and siRNA-guided DNA methylation. The enrichment of 24-nt siRNAs is an important indicator of RdDM activity. We generated and characterized genome-wide siRNA profiles for immature and ripe strawberry fruits (Fig. 5) and found that the number of siRNA clusters decreased during ripening. Further, the genomic regions that displayed reduced DNA methylation (hypo-DMRs) also displayed decreased siRNA enrichment during ripening (Fig. 5). Our siRNA analysis provides strong evidence for the diminished RdDM activity during strawberry ripening and also further supports our model that weakened $\mathrm{RdDM}$ contributes to the ripeninginduced DNA hypomethylation. RdDM has been extensively studied in Arabidopsis; however, a regulatory role for RdDM in plant development has not been established. Here, our study suggests a novel function of $\mathrm{RdDM}$ in the regulation of non-climacteric fruit ripening. In addition, strawberry CMT3 was downregulated during strawberry ripening, which could also contribute to the observed DNA hypomethylation.

We found that DNA hypomethylation and siRNA reduction preferentially occurred in the $5^{\prime}$ - and 
3 '-regulatory regions of genes (Fig. $5 \mathrm{~d}$ ), suggesting that RdDM-mediated DNA methylation regulates gene expression during strawberry ripening. Consistent with this hypothesis, genes involved in ripening-related processes, such as photosynthesis, cell wall biosynthesis, ABA biosynthesis, and fruit coloration, showed altered expression and DNA hypomethylation in their promoters during ripening. Interestingly, DNA hypomethylation did not always correlate with gene activation, but was also associated with gene repression during strawberry ripening. This is especially evident for photosynthesis and cell wall synthesis-related genes, which are no longer needed in ripe fruits and must be repressed so that they do not interfere with ripening. Ripening-associated DNA hypomethylation and gene repression were also observed in tomato, where mutations in the DNA demethylase SIDML2 prevented the DNA hypomethylation and consequently the repression of photosynthesis and cell wall synthesis-related genes was obstructed [14]. How promoter DNA methylation may help activate genes is not known. However, it is clear that although many transcription factors are sensitive to DNA methylation, some actually prefer binding to methylated motifs [27] and thus may activate the methylated genes. In addition, it is possible that the DNA binding of some transcriptional repressors may be sensitive to DNA methylation, and thus, loss of methylation would allow the repressors to reduce gene expression.

\section{Conclusions}

Our work suggests that DNA hypomethylation regulates the ripening of the non-climacteric strawberry fruit, as it does in the climacteric tomato fruit. Distinct mechanisms underlie the reduced levels of DNA methylation during strawberry and tomato ripening: diminished RdDM-mediated methylation versus increased DNA demethylation, respectively. DNA hypomethylation during ripening is associated with the altered expression of hundreds of ripening-related genes, suggesting that $\mathrm{RdDM}$ regulation of a genetic program contributes to fruit ripening.

\section{Methods}

\section{Plant materials}

Strawberries (Fragaria $\times$ ananassa Duch. cv. Hongjia) were grown in the scientific research innovation base at Zhejiang Academy of Agricultural Science at Haining (Zhejiang, China). Fruits at three developmental stages (Fa1, green stage, approximately 20 days after bloom (DAB); Fa2, intermediate red stage, $30 \mathrm{DAB} ; \mathrm{Fa} 3$, full red stage, $35 \mathrm{DAB}$ ) were harvested (Fig. 1a). After being transferred to the lab on the day of harvest, fruits with uniform size and free of visible defects were selected. Two biological replicates of 8-12 fruits each were used for each developmental stage.
For DNA methylation inhibitor treatment, "Si gongzhu" strawberry fruits were used. In the treatment, $20 \mathrm{mM}$ 5-azacytidine (Sigma) dissolved in $\mathrm{ddH}_{2} \mathrm{O}$ with $0.01 \%$ Triton X-100 was directly sprayed on the fruits. The treatment was performed on Jun. 8, 13, and 18. The samples were pictured on Jun. 23.

\section{Methylation-sensitive PCR}

Genomic DNA (100 ng) was digested with McrBC for $12 \mathrm{~h}$ according to the manufacturer's instructions. Digestion without GTP was used as negative control. After enzyme inactivation at $65^{\circ} \mathrm{C}$ for $20 \mathrm{~min}, 10 \%$ of digested DNA was used for each PCR reaction for Quantitative Real-time PCR. The primer sequence is included in Additional file 8: Table S7.

\section{Whole genome bisulfite sequencing and data analysis}

Genomic DNA was extracted from leaves and fruits using a DNeasy Plant Maxi Kit (Qiagen). The samples were sequenced at the Genomics Core Facility of the Shanghai Centre for Plant Stress Biology, Chinese Academy of Sciences, using Illumina HiSeq2500. The libraries of whole-genome bisulfite sequencing were prepared using NEBNext Ultra II DNA Library Prep Kit for Illumina (New England Biolabs) and Epitect Plus DNA Bisulfite Kit (Qiagen). Briefly, $1 \mu \mathrm{g}$ of genomic DNA was sonicated into 200 500 bp fragments on a Covaris M220. The fragmented DNA was subjected to end repair, A-tailing, and adaptor ligation following the manufacturer's instructions of the NEBNext Ultra II DNA Library Prep Kit. The adapter-ligated products were then treated with sodium bisulfite on a thermocycler using Epitect Plus DNA Bisulfite Kit (Qiagen) with the following program: $95^{\circ} \mathrm{C} 5 \mathrm{~min}, 60^{\circ} \mathrm{C} 25 \mathrm{~min}, 95^{\circ} \mathrm{C} 5$ $\min , 60^{\circ} \mathrm{C} 85 \mathrm{~min}, 95^{\circ} \mathrm{C} 5 \mathrm{~min}, 60^{\circ} \mathrm{C} 175 \mathrm{~min}$, and $3 \times\left(95^{\circ} \mathrm{C}\right.$ $5 \mathrm{~min}, 60^{\circ} \mathrm{C} 180 \mathrm{~min}$ ), hold at $20^{\circ} \mathrm{C}$. The BS-treated DNA was then cleaned up using Epitect Plus DNA Bisulfite Kit (Qiagen) and PCR-amplified using the KAPA HiFi Hotstart ReadyMix for 6 cycles. The amplified libraries were finally cleaned up using magnetic beads from Vazyme. The libraries were sequenced on a HiSeq2500 (Illumina) in paired-end 125 bp mode using the HiSeq PE Cluster Kit v4 (Illumina) and HiSeq SBS Kit v4 (250 cycles) (Illumina) following the manufacturer's instructions at Core Facility for Genomics of Shanghai Center for Plant Stress Biology.

For data analysis, paired-end sequencing reads were first trimmed with Trimmomatic [28] for removal of Illumina adapters and low-quality bases (quality score $<20$ ). The cleaned reads were then aligned to Fragaria vesca genome (Fragaria vesca v1.1, https://phytozome.jgi.doe.gov/pz/portal.html\#!linfo?alias=Org_Fvesca) using bsmap-2.87 [29] with default settings. Methylation ratio was extracted with methratio.py (a script included in bsmap-2.87). Properly mapped paired-end reads whose SAM flags equal 83 and 163 , or 99 and 147, were used by setting options $-\mathrm{u}$ and $-\mathrm{p}$ in methratio.py. DMCs were defined using Fisher's exact test 
$(p<0.05)$. DMRs were identified as previously described [30] with minor modifications. In brief, only cytosines with a depth of at least four in all libraries were considered. A sliding-window approach with window size 200-bp and step size 50-bp was used to identify DMRs. Fisher's exact test was performed for methylated vs. unmethylated cytosines within each window. False discovery rates (FDRs) were estimated using a Benjamini-Hochberg adjusted $P$ value. Windows with FDR $<0.05$ were defined as candidates for further analysis. DMRs were then adjusted by merging the candidates in all three contexts and shrinking to the first and last differentially methylated cytosines (DMCs). Final DMRs were filtered with combined criteria: number of DMC > 3 and mean methylation difference > 0.15 . To incorporate biological replicates into analysis, we calculated robust index as the formula for each DMR to measure the repeatability between replicates.

$$
\text { Robust index }=\frac{\left|\log _{2} F C_{1}-\log _{2} F C_{2}\right|}{\left|\log _{2} F C_{1}+\log _{2} F C_{2}\right|}
$$

$\mathrm{FC}_{1}$ and $\mathrm{FC}_{2}$ represent differential methylation fold change in replicate 1 and replicate 2, respectively. DMRs with lower robust index are more credible in terms of differential methylation. Robust index for DMRs with low depth in individual replicates were assigned as NA. We ranked the DMRs by robust index (Additional file 3: Table S2) and re-analyzed both top 500 and least 500 credible hypo DMRs respectively. As shown in Additional file 2: Figure S7, the analysis of both groups of DMRs supports major conclusions in the main text, including gradual decrease in DNA methylation during ripening (Additional file 2: Figure S7a), enrichment of DMRs in promoter regions (Additional file 2: Figure S7b), and decreased siRNA accumulation in hypo-DMRs (Additional file 2: Figure S7c). Principal component analysis (PCA) was used to cluster the methylation patterns of fruits and leaves in DMRs into low dimensions. Integrated Genome Browser (IGB) [31] was used to visualize the DNA methylation data. DMR-associated genes were defined as genes with the closest DMR located within $2 \mathrm{~kb}$ upstream of the transcription start site (TSS) and $2 \mathrm{~kb}$ downstream of the transcription end site (TES)).

\section{RNA sequencing and data analysis}

Total RNA was extracted with TRIzol reagent (Ambion) from fruits. For reverse transcription, $1 \mu \mathrm{g}$ of RNA and oligo dT primers were used to synthesize cDNA in a $20-\mu \mathrm{L}$ reaction using the qScript cDNA SuperMix kit (Quanta). For RNA-seq, the libraries were constructed and sequenced at the Genomics Core Facility of the Shanghai Centre for Plant Stress Biology, Chinese Academy of Sciences, using an Illumina HiSeq2500.

For data analysis, paired-end reads were aligned to Fragaria vesca genome (Fragaria vesca v1.1, https://
phytozome.jgi.doe.gov/pz/portal.html\#!info?alias=Org_Fvesca) using STAR [32] with default parameters. Uniquely mapped reads with MAPQ $>20$ were collected for further analysis. FeatureCounts [33] was used to count the mapped fragments for each gene. DESeq [34] was used to detect differentially expressed genes. To assign putative functions of genes in Fragaria vesca, gene orthologs provided by Phytozome [35] (https://phytozome.jgi.doe.gov/pz/portal.html) were collected and the function of corresponding Arabidopsis ortholog was assigned. Fisher's exact test was used to determine whether the gene set is significantly enriched in a specific GO term. Enriched GO terms were defined base on combined criteria: $\mid \log _{2}$ Fold change $\mid>1$ and $P$ value $<0.05$. To valid the gene expression changes during ripening, published datasets (accession number PRJNA394190 (https:// www.ncbi.nlm.nih.gov/bioproject/394190)) which characterized the transcriptome change during strawberry ripening were downloaded and processed.

\section{Small-RNA sequencing and data analysis}

Total RNA was extracted with TRIzol reagent (Ambion) from fruits. Total RNA was then separated on denaturing polyacrylamide gels, and $<100$-nt fractions were cut out and purified for standard small RNA library preparation. For small-RNA sequencing, the libraries were constructed and sequenced at the Genomics Core Facility of the Shanghai Centre for Plant Stress Biology, Chinese Academy of Sciences, using an Illumina HiSeq2500.

Sequenced reads were trimmed with Trimmomatic [28] to remove Illumina adapters and low-quality bases (quality score $<20$ ). Reads with length $<18 \mathrm{bp}$ and length $>30$ bp were discarded. Cleaned 24-nt reads were mapped to $F$. vesca genome and defined to siRNA cluster with Shortstack [36]. The mapped reads were normalized by total cleaned reads for further analysis.

\section{Phylogenetic analysis}

The orthologs of DNA methyltransferase, DNA demethylase, and gene involved in RdDM pathway annotated in Phytozome were used to build a Neighbor-Joining phylogenetic trees with MEGA [37].

\section{Virus-induced gene silencing (VIGS)}

The TRV vectors pTRV1 (pYL192) and pTRV2 (pYL156) for gene silencing have been described by Liu et al. [38], and the treatment protocol was as described by Birch-Smith et al. [39]. pTRV2 vector was ligated with PCR fragment of $F v A G O 4$ and then transformed into A. tumefaciens GV3101 strain. The Agrobacterium culture (grown in $25 \mathrm{mg} / \mathrm{L}$ Rifampicin and $50 \mathrm{mg} / \mathrm{L}$ Kanamycin overnight culture) was transformed with pTRV1, pTRV2 and pTRV2 derivative pTRV2-FvAGO4, respectively. Agrobacterium culture was infiltrated into fruit pedicel of strawberry. 


\section{Additional files}

Additional file 1: Table S1. Mapping statistics of bisulfite sequencing, RNA sequencing, and small-RNA sequencing. (XLSX $19 \mathrm{~kb}$ )

Additional file 2: Figure S1. The strawberry methylomes. Figure S2 Methylomes of fruits at different stages. Figure S3 Heatmaps showing DNA methylation changes (Fa3-Fa1) across hyper-DMR-associated genes. Figure S4 Expression of genes involved in DNA demethylation. Figure S5 Analyses of siRNAs in strawberry fruits. Figure S6 Association between gene expression and DNA methylation during ripening. Figure S7 Repeatability between replicates using robust index. (DOCX $14141 \mathrm{~kb}$ )

Additional file 3: Table S2. Summary of 466 hyper DMRs and 2300 hypo DMRs in Fa3 compared to Fa1. (XLSX 283 kb)

Additional file 4: Table S3. Expression of genes involved in DNA methylation and demethylation during ripening process. (XLSX 17 kb)

Additional file 5: Table S4. Genomic coordinates of 370,630 24-nt siRNA clusters in strawberry fruits. (XLSX $7562 \mathrm{~kb}$ )

Additional file 6: Table S5. Summary of 2316 genes with differential expression and differential methylation levels in Fa3 compared to Fa1. (XLSX $317 \mathrm{~kb}$ )

Additional file 7: Table S6. Enriched GO terms of 555 hypomethylated down-DEGs, 367 hypomethylated up-DEGs, 862 non-hypomethylated down-DEGs, and 532 non-hypomethylated up-DEGs. (XLSX 36 kb)

Additional file 8: Table S7. Primers for q-PCR and VIGS. (XLSX 9 kb)

Additional file 9: Review history. (DOCX $57 \mathrm{~kb}$ )

\section{Abbreviations}

5'-Aza: 5-Azacytidine; ABA: Abscisic acid; AGO4/6: Argonaute 4/6; At: Arabidopsis thaliana; CMT2: Chromomethylase 2; CMT3: Chromomethylase 3; CNR: Colorless non-ripening; DCLs: Dicer-likes; DEG: Differentially expressed genes; DMCs: Differentially methylated cytosines; DMRs: Differentially methylated regions; DRMs: Domains rearranged methylases; FDR: False discovery rates; Fv: Fragaria vesca; Fa: Fragaria $x$ ananassa; VIGS: Virus-induced gene silencing; GO: Gene ontology; IDM: Increase of DNA methylation; MET1: Methyltransferase 1: NCED: 9-cis-epoxycarotenoid dioxygenase; PCA: Principal component analysis; Pol IV: RNA polymerase IV; Pol V: RNA polymerase $\mathrm{V}$; RdDM: RNA-directed DNA methylation; ROS1: Repressor of silencing 1; siRNA: Small interfering RNA; SI: Solanum lycopersicum; TE: Transposable element; MAPQ: Mapping quality; TSS: Transcriptional start sites; TES: Transcriptional end sites; mC: DNA methylation of all cytosines sites; mCG: DNA methylation of CG sites; mCHG: DNA methylation of CHG sites; $\mathrm{mCHH}$ : DNA methylation of $\mathrm{CHH}$ sites; FPKM: Fragments per kilobase million; Chr: Chromosome

\section{Acknowledgements}

This work was supported by National Key R\&D Program of China (2016YFD0400100) and Strategic Priority Research Program of the Chinese Academy of Sciences (Grant No. XDB27040000) (to Z. L.).

\section{Review history}

The review history is available as Additional file 9.

\section{Funding}

This work was supported by the Chinese Academy of Sciences (to Z. L.).

\section{Availability of data and materials}

The data generated in this study have been deposited in Gene Expression Omnibus under the accession number GSE113084. To valid the gene expression changes during ripening, published datasets (accession number PRJNA394190 (https://www.ncbi.nlm.nih.gov/bioproject/394190)) which characterized the transcriptome change during strawberry ripening were downloaded and processed [40].

\section{Authors' contributions}

$J C, Q N, Z Y, J-K Z$, and ZL designed the study and interpreted the data. JC and

ZL wrote the manuscript. JC did the bioinformatics analysis. QN, BZ, and KC prepared the fruit samples for the experiments. $\mathrm{HZ}$ performed the sequencing experiments. All authors read and approved the final manuscript.

Ethics approval and consent to participate

Not applicable.

\section{Consent for publication}

Not applicable.

\section{Competing interests}

The authors declare that they have no competing interests.

\section{Publisher's Note}

Springer Nature remains neutral with regard to jurisdictional claims in published maps and institutional affiliations.

\section{Author details}

${ }^{1}$ National Key Laboratory of Plant Molecular Genetics, CAS Center of Excellence in Molecular Plant Sciences, Institute of Plant Physiology and Ecology, Shanghai Institutes for Biological Sciences, Chinese Academy of Sciences, Shanghai 200032, China. ${ }^{2}$ University of the Chinese Academy of Sciences, Beijing 100049, China. ${ }^{3}$ Shanghai Center for Plant Stress Biology, National Key Laboratory of Plant Molecular Genetics, Center of Excellence in Molecular Plant Sciences, Shanghai Institutes for Biological Sciences, Chinese Academy of Sciences, Shanghai 200032, China. ${ }^{4}$ Laboratory of Fruit Quality Biology/Zhejiang Provincial Key Laboratory of Horticultural Plant Integrative Biology, Zhejiang University, Zijingang Campus, Hangzhou 310058, China. ${ }^{5}$ Horticultural Department, Heilongjiang Academy of Agricultural Sciences, Harbin, China. ${ }^{6}$ Department of Horticulture and Landscape Architecture, Purdue University, West Lafayette, IN 47907, USA.

Received: 4 September 2018 Accepted: 15 November 2018

Published online: 04 December 2018

\section{References}

1. Zhu JK. Active DNA demethylation mediated by DNA glycosylases. Annu Rev Genet. 2009;43:143-66

2. Law JA, Jacobsen SE. Establishing, maintaining and modifying DNA methylation patterns in plants and animals. Nat Rev Genet. 2010;11:204-20.

3. Zemach A, Kim MY, Hsieh PH, Coleman-Derr D, Eshed-Williams L, Thao K, Harmer SL, Zilberman D. The Arabidopsis nucleosome remodeler DDM1 allows DNA methyltransferases to access $\mathrm{H1}$-containing heterochromatin. Cell. 2013:153:193-205.

4. Matzke MA, Kanno T, Matzke AJ. RNA-directed DNA methylation: the evolution of a complex epigenetic pathway in flowering plants. Annu Rev Plant Biol. 2015;66:243-67.

5. Matzke MA, Mosher RA. RNA-directed DNA methylation: an epigenetic pathway of increasing complexity. Nat Rev Genet. 2014;15:394-408.

6. Zhang $\mathrm{H}$, Zhu JK. Active DNA demethylation in plants and animals. Cold Spring Harb Symp Quant Biol. 2012;77:161-73.

7. Klee HJ, Giovannoni JJ. Genetics and control of tomato fruit ripening and quality attributes. Annu Rev Genet. 2011:45:41-59.

8. Jia HF, Chai YM, Li CL, Lu D, Luo JJ, Qin L, Shen YY. Abscisic acid plays an important role in the regulation of strawberry fruit ripening. Plant Physiol. 2011;157:188-99.

9. Manning K, Tor M, Poole M, Hong Y, Thompson AJ, King GJ, Giovannoni J, Seymour GB. A naturally occurring epigenetic mutation in a gene encoding an SBP-box transcription factor inhibits tomato fruit ripening. Nat Genet. 2006;38:948-52.

10. El-Sharkawy I, Liang D, Xu KN. Transcriptome analysis of an apple (Malus x domestica) yellow fruit somatic mutation identifies a gene network module highly associated with anthocyanin and epigenetic regulation. J Exp Bot. 2015;66:7359-76

11. Telias A, Kui LW, Stevenson DE, Cooney JM, Hellens RP, Allan AC, Hoover EE, Bradeen JM. Apple skin patterning is associated with differential expression of MYB10. BMC Plant Biol. 2011;11:93.

12. Zhong SL, Fei ZJ, Chen YR, Zheng Y, Huang MY, Vrebalov J, McQuinn R, Gapper N, Liu B, Xiang J, et al. Single-base resolution methylomes of tomato fruit development reveal epigenome modifications associated with ripening. Nat Biotechnol. 2013;31:154-9. 
13. Liu RE, How-Kit A, Stammitti L, Teyssier E, Rolin D, Mortain-Bertrand A, Halle S, Liu MC, Kong JH, Wu CQ, et al. A DEMETER-like DNA demethylase governs tomato fruit ripening. Proc Natl Acad Sci U S A. 2015;112:10804-9.

14. Lang ZB, Wang YH, Tang K, Tang DG, Datsenka T, Cheng JF, Zhang YJ, Handa AK, Zhu JK. Critical roles of DNA demethylation in the activation of ripening-induced genes and inhibition of ripening-repressed genes in tomato fruit. Proc Natl Acad Sci U S A. 2017;114:E4511-9.

15. Shulaev V, Sargent DJ, Crowhurst RN, Mockler TC, Folkerts O, Delcher AL, Jaiswal P, Mockaitis K, Liston A, Mane SP, et al. The genome of woodland strawberry (Fragaria vesca). Nat Genet. 2011;43:109-16.

16. Bibikova M, Fan JB. Genome-wide DNA methylation profiling. Wiley Interdiscip Rev Syst Biol Med. 2010;2:210-23.

17. Niederhuth CE, Bewick AJ, Ji LX, Alabady MS, Kim KD, Li Q, Rohr NA Rambani A, Burke JM, Udall JA, et al. Widespread natural variation of DNA methylation within angiosperms. Genome Biol. 2016;17:194

18. Rousseau-Gueutin M, Lerceteau-Kohler E, Barrot L, Sargent DJ, Monfort A, Simpson D, Arus P, Guerin G, Denoyes-Rothan B. Comparative genetic mapping between octoploid and diploid Fragaria species reveals a high level of colinearity between their genomes and the essentially disomic behavior of the cultivated octoploid strawberry. Genetics. 2008;179:2045-60.

19. Lister R, O'Malley RC, Tonti-Filippini J, Gregory BD, Berry CC, Millar AH, Ecker $J R$. Highly integrated single-base resolution maps of the epigenome in Arabidopsis. Cell. 2008;133:523-36.

20. Price AL, Jones NC, Pevzner PA. De novo identification of repeat families in large genomes. Bioinformatics. 2005;21:1351-8.

21. Underwood CJ, Henderson IR, Martienssen RA. Genetic and epigenetic variation of transposable elements in Arabidopsis. Curr Opin Plant Biol. 2017;36:135-41.

22. Lang ZB, Lei MG, Wang XG, Tang K, Miki D, Zhang HM, Mangrauthia SK, Liu WS, Nie WF, Ma GJ, et al. The methyl-CpG-binding protein MBD7 facilitates active DNA demethylation to limit DNA hyper-methylation and transcriptional gene silencing. Mol Cell. 2015;57:971-83.

23. Qian WQ, Miki D, Zhang H, Liu YH, Zhang X, Tang K, Kan YC, La HG, Li XJ, Li SF, et al. A histone acetyltransferase regulates active DNA demethylation in Arabidopsis. Science. 2012;336:1445-8.

24. Mi SJ, Cai T, Hu YG, Chen Y, Hodges E, Ni FR, Wu L, Li S, Zhou H, Long CZ, et al. Sorting of small RNAs into Arabidopsis argonaute complexes is directed by the 5 ' terminal nucleotide. Cell. 2008;133:116-27.

25. Song C, Ring L, Hoffmann T, Huang FC, Slovin J, Schwab W. Acylphloroglucinol biosynthesis in strawberry fruit. Plant Physiol. 2015;169:1656-70.

26. Bottcher C, Burbidge CA, Boss PK, Davies C. Changes in transcription of cytokinin metabolism and signalling genes in grape (Vitis vinifera L.) berries are associated with the ripening-related increase in isopentenyladenine. BMC Plant Biol. 2015;15:223.

27. O'Malley RC, Huang SC, Song L, Lewsey MG, Bartlett A, Nery JR, Galli M, Gallavotti A, Ecker JR. Cistrome and epicistrome features shape the regulatory DNA landscape. Cell. 2016;166:1598.

28. Bolger AM, Lohse M, Usadel B. Trimmomatic: a flexible trimmer for Illumina sequence data. Bioinformatics. 2014:30:2114-20.

29. Xi Y, Li W. BSMAP: whole genome bisulfite sequence MAPping program. BMC Bioinformatics. 2009:10:232.

30. Ausin I, Greenberg MVC, Simanshu DK, Hale CJ, Vashisht AA, Simon SA, Lee TF, Feng SH, Espanola SD, Meyers BC, et al. INVOLVED IN DE NOVO 2containing complex involved in RNA-directed DNA methylation in Arabidopsis. Proc Natl Acad Sci U S A. 2012;109:8374-81.

31. Nicol JW, Helt GA, Blanchard SG Jr, Raja A, Loraine AE. The Integrated Genome Browser: free software for distribution and exploration of genomescale datasets. Bioinformatics. 2009;25:2730-1.

32. Dobin A, Davis CA, Schlesinger F, Drenkow J, Zaleski C, Jha S, Batut P, Chaisson M, Gingeras TR. STAR: ultrafast universal RNA-seq aligner. Bioinformatics. 2013;29:15-21.

33. Liao Y, Smyth GK, Shi W. featureCounts: an efficient general purpose program for assigning sequence reads to genomic features. Bioinformatics. 2014;30:923-30.

34. Anders S, Huber W. Differential expression analysis for sequence count data. Genome Biol. 2010;11:R106.

35. Goodstein DM, Shu S, Howson R, Neupane R, Hayes RD, Fazo J, Mitros T, Dirks W, Hellsten U, Putnam N, Rokhsar DS. Phytozome: a comparative platform for green plant genomics. Nucleic Acids Res. 2012;40:D1178-86.

36. Axtell MJ. ShortStack: comprehensive annotation and quantification of small RNA genes. RNA. 2013;19:740-51.
37. Kumar S, Stecher G, Tamura K. MEGA7: molecular evolutionary genetics analysis version 7.0 for bigger datasets. Mol Biol Evol. 2016:33:1870-4.

38. Liu Y, Schiff M, Dinesh-Kumar SP. Virus-induced gene silencing in tomato. Plant J. 2002:31:777-86.

39. Burch-Smith TM, Anderson JC, Martin GB, Dinesh-Kumar SP. Applications and advantages of virus-induced gene silencing for gene function studies in plants. Plant J. 2004;39:734-46.

40. Hu P, Li G, Zhao X, Zhao F, Li L, Zhou H. Transcriptome profiling by RNA-Seq reveals differentially expressed genes related to fruit development and ripening characteristics in strawberries (Fragaria x ananassa). PeerJ. 2018;6:e4976.

\section{Ready to submit your research? Choose BMC and benefit from:}

- fast, convenient online submission

- thorough peer review by experienced researchers in your field

- rapid publication on acceptance

- support for research data, including large and complex data types

- gold Open Access which fosters wider collaboration and increased citations

- maximum visibility for your research: over $100 \mathrm{M}$ website views per year

At BMC, research is always in progress.

Learn more biomedcentral.com/submissions 